\title{
根系隔离条件下的谷子亲缘识别
}

\author{
陈青青 1 李德志 $1,2,3^{*}$ \\ 1华东师范大学生态与环境科学学院, 上海 200241; ${ }^{2}$ 华东师范大学上海市城市化生态过程与生态恢复重点实验室, 上海 $200241 ;{ }^{3}$ 华东师范大学浙江 \\ 天童森林生态系统国家野外科学观测研究站, 上海 200241
}

摘 要 在根系隔离情况下, 通过研究邻株身份(亲缘株、非亲缘株、陌生株)及其与种植密度(高、低)和土壤养分水平(高、 低)交互作用对谷子(Setaria italica)形态学特征和生物量分配的影响, 探索谷子地上部分是否能够识别亲缘邻株, 以及谷子的 这种亲缘识别能力对环境因子如何响应。结果显示: 1)亲缘组谷子叶生物量分配显著降低, 茎秆显著增粗, 暗示着亲缘组谷子 植株间减少竞争, 并增强对当地多风气候的适应。而非亲缘组谷子叶生物量分配显著增加, 表明非亲缘组谷子植株间竞争较 强。2)与非亲缘组相比, 陌生组谷子种子生物量分配显著增加, 株高显著减少, 表明陌生组谷子植株通过不对称竞争(与邻株 糜(Panicum miliaceum)植株相比, 株高显著增加), 进一步限制邻株(糜)生长, 从而增强竞争能力, 同时, 将更多的生物量投资 于繁殖, 增加适合度。3)在高密度种植条件下, 谷子茎生物量和叶生物量分配在各邻株身份处理间无显著差异, 而在低密度种 植条件下, 与非亲缘组相比, 亲缘组谷子茎生物量显著增加, 叶生物量分配显著减小; 随着种植间距的增大(种植密度减小), 亲 缘组谷子叶生物量分配显著减少, 而非亲缘组和陌生组叶生物量分配在高、低种植密度条件下无显著差异。4)在低土壤养分条 件下, 亲缘组和非亲缘组谷子叶生物量分配无显著差异, 前者穗长显著小于后者, 而在高土壤养分条件下, 亲缘组谷子叶生物 量分配显著小于非亲缘组, 前者穗长显著大于后者。结果表明, 在根系隔离的情况下, 谷子能够识别亲缘邻株, 且谷子地上部分 竞争信号在亲缘识别过程中扮演重要角色。较低种植密度和较高土壤养分水平有利于谷子亲缘识别能力的表达。

关键词 谷子, 竞争, 生物量分配, 亲缘识别, 亲缘选择, 根系隔离

引用格式: 陈青青, 李德志 (2015). 根系隔离条件下的谷子亲缘识别. 植物生态学报, 39, 1188-1197. doi: 10.17521/cjpe.2015.0115

\section{Kin recognition in Setaria italica under the condition of root segregation}

\section{CHEN Qing-Qing ${ }^{1}$ and LI De-Zhi, ${ }^{1,2,3 *}$}

${ }^{1}$ School of Ecological and Environmental Science, East China Normal University, Shanghai 200241, China; ${ }^{2}$ Key Laboratory of Urbanization and Ecological Restoration of Shanghai, Shanghai 200241, China; and ${ }^{3}$ National Field Observation and Research Station in Tiantong Forest Ecosystem of Zhejiang, Shanghai 200241, China

\section{Abstract}

Aims Kin recognition may play an important part in the performance and productivity of crop plants. However, so far, little is known about whether crop plants can recognize their kin neighbors. The aim of this study was to explore kin recognition in Setaria italica, and its responses to changes in environmental and biological conditions. Methods A field experiment was conducted in the suburb of Shanghai. Setaria italica grew with different neighbors (kin, non-kin and strangers), under the condition of root segregation and different plant densities (high and low) and soil nutrient levels (high and low), respectively. We investigated how neighbor identity and its interactions with plant density and soil nutrient level affected the morphology and biomass allocation of S. italica.

Important findings Under the condition of root segregation, 1) Leaf biomass allocation and stem diameter of plants in the kin groups significantly decreased and increased, respectively, suggesting that plants of $S$. italica in the kin groups reduced inter-individual competition, and adapted to the local windy climate. 2) Compared with the non-kin groups, plants in the stranger groups significantly increased the biomass allocation to seeds, while plant height decreased significantly, suggesting that the plants of S. italica in the stranger groups may reduce the growth of their neighbors due to asymmetric competition ( $S$. italica significantly increased height compared with the neighboring plants, Panicum miliaceum). Therefore, the S. italic plants in the stranger groups allocated more biomass to reproduction and increased fitness than those in non-kin groups. 3) Under the condition of high plant density, no significant differences were found in stem biomass and leaf biomass allocation of plants among 
different neighbor identity treatments. While under the condition of low plant density, compared with the non-kin groups, biomass allocation to stem and leaf in the kin groups significantly increased and decreased, respectively. As the plant density decreased, plants in the kin groups decreased leaf biomass allocation significantly, while plants in the non-kin and stranger groups did not show such a response. 4) Under the condition of low soil nutrient level, no significant difference was found in leaf biomass allocation between the kin and non-kin groups, while the ear length of plants in the kin groups decreased significantly. Under the condition of high soil nutrient level, the biomass allocated to leaves in the kin groups decreased significantly, while ear length increased significantly compared with the non-kin groups. Therefore, under the condition of root segregation, plants of $S$. italica showed the ability to recognize their kin neighbors, and the aboveground competitive cues may play important roles in the course of kin recognition in S. italica. Lower plant density and higher soil nutrient level may facilitate the ability of kin recognition in S. italica.

Key words Setaria italica, competition, biomass allocation, kin recognition, kin selection, root segregation

Citation: Chen QQ, Li DZ (2015). Kin recognition in Setaria italica under the condition of root segregation. Chinese Journal of Plant Ecology, 39, 1188-1197. doi: 10.17521/cjpe.2015.0115

植物间的正相互作用(互助)和负相互作用(竞 争)是影响群落结构、多样性和动态的一个重要过程 (Callaway, 2007; Brooker et al., 2008)。一些植物由于 自交、繁殖体传播距离有限、营养繁殖或人工选择 等(如作物、经济林等), 更有可能与亲缘近的植株为 邻。生态位分化理论预测, 亲缘上相近的植株间, 由 于更多的生态位重叠, 将产生更激烈的竞争 (Silvertown, 2004), 但支持亲缘间(包括在自交和异 交的有性生殖过程中, 由同一个母本产生的后代, 下同)的竞争大于非亲缘(同一种植物不同母本产生 的后代, 下同)的证据十分有限(Cheplick, 1992)。相 反, 有研究发现, 当一些植物与亲缘近的植株为邻 时, 生长更好(Willson et al., 1987), 适合度增加 (Tonsor, 1989)。植物的这种行为可以用亲缘选择理 论来解释, 基因上相似度高的个体间可以通过相互 合作减少用于竞争的资源消耗，从而将更多的资源 投资于繁殖并增加适合度(Hamilton，1964a，1964b; Axelrod \& Hamilton, 1981)。当植物的邻居为亲缘株 时, 亲缘选择和生态位分化可能同时起作用, 且两 者作用于同一个过程(File et al., 2012; Dudley et al., 2013)。

Dudley和File等(2007)报道了一年生植物Cakile edentula可能通过根系的相互作用识别亲缘邻株, 在根系接触的情况下, 和与非亲缘株为邻相比, 与 亲缘株为邻的植株细根生物量显著减少, 即亲缘邻 株间减少了根系竞争。该论文发表后, 植物亲缘识 别现象得到了较多的关注、讨论和研究(Biedrzycki \& Bais, 2010; Chen et al., 2012; File et al., 2012; Dudley et al., 2013; 李洁等, 2014)。目前, 有关植物
亲缘识别的一些研究关注邻株身份如何影响基于植 物繁殖表现的适合度(Mohana et al., 2001; Milla et al., 2009, 2012; Bhatt et al., 2011; Biernaskie, 2011), 另一些关注不同邻株身份对竞争性状及其生物量分 配的影响(Dudley \& File, 2007; Murphy \& Dudley, 2009; Biedrzycki et al., 2010; Bhatt et al., 2011; Lepik et al., 2012; Dudley et al., 2013; Fang et al., 2013; Mercer \& Eppley, 2014; Semchenko et al., 2014)。另 外, 地下部分竞争信号在植物身份识别过程中所扮 演的角色得到较多的关注(Dudley \& File, 2007; Murphy \& Dudley, 2009; Biedrzycki et al., 2010; Bhatt et al., 2011; Lepik et al., 2012; Dudley et al., 2013; Fang et al., 2013; Mercer \& Eppley, 2014; Semchenko et al., 2014), 少量的研究表明地上部分 竞争信号在植物身份识别过程中也扮演着一定的角 色(Crepy \& Casal, 2015)。迄今为止, 植物亲缘识别 研究还没有一致的结论。但某些植物在一定的环境 下能够识别亲缘邻株已是不争的事实(Hess \& de Kroon, 2007)。农作物群体大多是在人为经营、控制 下形成的单一品种的种群, 因此很有可能与亲缘近 的植株为邻。植物亲缘识别可能对农作物生长和产 量有重要影响(Biedrzycki \& Bais, 2010; Chen et al., 2012; Bais, 2015; Crepy \& Casal, 2015), 然而, 目前 相关的报道甚少。

胁迫梯度假说预测: 随着环境胁迫的增加, 植 物间竞争将减少, 而互助将增加, 并占主导作用, 即一些植物可以缓和潜在的环境胁迫, 从而为其他 植物创造更适宜的生存环境(Bertness \& Callaway, 1994; Maestre et al., 2009)。迄今为止, 植物间的互 
助研究较多关注植物种间的互助, 如个体较大的植 物通过提供较好的林下遮阴环境为新物种的迁入和 定居提供便利(Farrer \& Goldberg, 2011)。近年来, 胁 迫梯度假说在一些植物种内的植株间也得到证实 (Fajardo \& McIntire, 2011; Farrer \& Goldberg, 2011; Cavieres \& Penaloza, 2012; Clara Castellanos et al., 2014)。在严重胁迫的环境条件下, 甚至在中度胁迫 时, Nothofagus pumilio同种植株间也表现出互助 (Fajardo \& McIntire, 2011)。胁迫梯度假说是否也适 用于亲缘植株间? 或者如其他学者所预测, 亲缘识 别可能只在较好的环境条件下表达, 而在恶劣的环 境条件下, 个体会尽最大可能增加自身的适合度, 而具有代价的利他行为可能是不利的(Pakkasmaa \& Laurila, 2004; Biedrzycki \& Bais, 2010)。

基于此, 本文以谷子“沈黄2号” (Setaria italica 'Qinhuang 2')为研究材料, 在根系隔离条件下, 通 过研究邻株身份及其与种植密度和土壤养分水平交 互作用对谷子形态学特征和生物量分配的影响, 探 索在地下部分竞争信号不存在的情况下, 谷子是否 能够识别亲缘邻株以及亲缘识别对环境因子如何 响应。

\section{1 材料和方法}

\section{1 材料和研究地概况}

谷子为禾本科一年生栽培草本粮食作物。高 0.1-1.2 m, 秆粗壮、分藥少, 长披针形叶片, 主营自 花授粉, 穗状圆雉花序, 长0.2-0.30 m。广泛栽培于 欧亚大陆的温带和热带。中国黄河中上游为主要栽 培区, 其他地区也有少量栽种。供试谷子品种为“沈 黄2号', 生育期约为 112 天。

糜(Panicum miliaceum)为禾本科一年生栽培草 本粮食作物。秆粗壮、直立, 高0.4-1.2 m, 单生或 少数从生, 有时有分枝, 叶片线形或线状披针形, 圆锥花序开展或较紧密, 成熟时下垂, 长0.1-0.3 $\mathrm{m}$ 。在亚洲、欧洲、美洲、非洲等温暖地区都有栽 培。我国西北、华北、西南、东北、华南以及华东 等地山区都有栽培。供试糜品种为“金糜1号”, 生育 期约为 105 天。

实验于2014年4月至11月在上海市闵行区马桥 镇韩湘蔬菜种植园内进行。实验期间该地区平均气 温 $25.27{ }^{\circ} \mathrm{C}$, 总降水757.56 mm, 盛行东南风, 平均2 $\min$ 的风速为 $1.90 \mathrm{~m} \cdot \mathrm{s}^{-1}$, 平均最大风速为 $2.45 \mathrm{~m} \cdot \mathrm{s}^{-1}$,
平均极大风速为 $4.59 \mathrm{~m} \cdot \mathrm{s}^{-1}$ (数据由上海市闵行区气 象局提供)。该种植园附近近年来均没有种植谷子和 糜。实验地约 $100 \mathrm{~m}^{2}$, 周围种植梨树。实验开始前 测量土壤全氮为 $3.54 \mathrm{mg} \cdot \mathrm{g}^{-1}$ 。

\section{2 方法}

\subsection{1 实验设计}

由于种植密度和土壤养分水平显著影响谷子 生长和产量(刘恩魁等, 2013; 杨艳君等, 2013), 本 实验设置了包括邻株身份(亲缘株、非亲缘株和陌生 株)、种植密度(高和低)、土壤养分水平(高和低) 3 个因素的交互处理实验。种植园内共设24个小区(图 1)。实验开始前先犁地, 使0-30 cm土层及整个实验 地土壤充分混匀。在平整的实验地上，每个小区按 长 $\times$ 宽 $\times$ 深 $=50 \mathrm{~cm} \times 50 \mathrm{~cm} \times 25 \mathrm{~cm}$ 挖坑, 挖出的土 捣碎, 充分混匀, 并装入相应规格的食用菌栽培袋 (高密度小区25袋，低密度小区9袋，栽培袋直径分 别为 $10 \mathrm{~cm}$ 和 $16.7 \mathrm{~cm}$, 长裁剪至 $28 \mathrm{~cm}$, 厚度 $<1$ $\mathrm{mm})$, 压实, 至 $25 \mathrm{~cm}$ 处, 用针在栽培袋底部及四周 扎孔, 利于多余水分及时溢出。装满土的栽培袋放 入各小区, 均匀紧密摆放, 剩余土壤回填到栽培袋 间隙。各小区间隔30-50 cm, 以减少干扰。其中亲 缘株种植、非亲缘株种植和陌生株种植小区各 8 个。 在各邻株身份处理中, 高密度和低密度小区各4个。 24 个小区中一半施肥、一半不施肥, 分别作为高、 低土壤养分处理。

\subsection{2 邻株身份}

实验中的亲缘株是指其种子来自同品种、同穗 的谷子, 非亲缘株是指其种子来自同品种、不同穗 的谷子, 陌生株是指有别于谷子的其他种类作物 (以糜作为陌生株)。谷穗于上一年收集自山西省长 治市的一处谷子种植区。挑选 4 个较大谷穗, 每个谷 穗作为两个亲缘株种植小区的种子库, 以此产生 4 个亲缘, 各 2 个重复的亲缘株种植小区。从上述同一 种植区内随机选出 30 个谷穗进行混合, 作为非亲缘 株种植小区的谷子种子库。陌生株种植小区采用谷 子和糜间作 (奇数株为谷子, 偶数株为糜, 见图1), 其中谷子的种子来自 30 个谷穗的混合种子库, 糜种 子来源于黑龙江省哈尔滨市的一处糜种植区的混合 种子。

\subsection{3 种植密度}

参考刘恩鬼等(2013)和杨艳君等(2013)提出的 最大谷子产量的最佳种植密度, 设定高密度为 100 


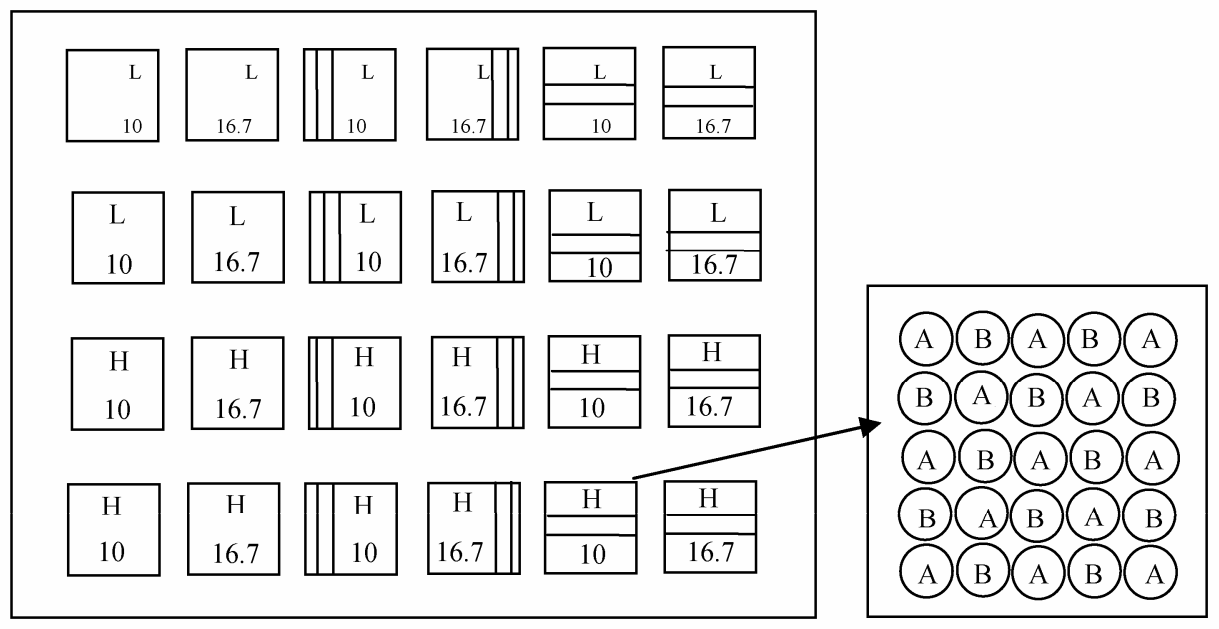

图1 实验方案示意图。空白框, 亲缘组; 坚条框, 非亲缘组; 横条框, 陌生组。 L, 低营养; H, 高营养。10和16.7, 种植间距 $(\mathrm{cm})$ 。 A, 混合谷子种子; B, 混合糜种子。

Fig. 1 Diagram of experimental design. Blank box, kin group; Vertical bars box, non-kin group; Stripes box, stranger group. H, high soil nutrient level; L, low soil nutrient level. 10 and 16.7, plant spacing $(\mathrm{cm})$. A, mixed seeds of Setaria italica; B, mixed seed of Panicum miliaceum.

ind. $\mathrm{m}^{-2}$, 即每小区 25 株, 植株间距 $10 \mathrm{~cm}$; 低密度 为 36 ind. $\mathrm{m}^{-2}$, 即每小区9株, 植株间距 $16.7 \mathrm{~cm}$ 。

\subsection{4 土壤养分}

参考刘斌等(2013)提出的谷子种植的最适宜氮 肥用量, 设定高土壤养分处理为每小区追施尿素6 $\mathrm{g}$, 即氮 $11.2 \mathrm{~g} \cdot \mathrm{m}^{-2}$ 。在谷子灌浆期至抽穗初期进行施 肥。施肥前先将尿素溶解于 $1.5 \mathrm{~L}$ 水中, 再均匀喷酒 到植株基部。对于不施肥处理的小区, 同时喷酒等 量的水。

\subsection{5 种植管理}

2014年5月29日进行穴播, 在每个栽培袋中央 处, 播2-3粒种子, 同时在实验地空白区播同穗、不 同穗的谷子种子和糜种子, 以备早期补苗。出苗两 个星期后, 实施间苗及补苗, 使每个栽培袋保留一 株长势一致的幼苗。7月7日进行高、低养分施肥处 理。在试验过程中, 定期观察并记录植株生长情况, 及时除去杂草。在土壤缺水时, 及时浇水。在种子 成熟前, 在实验地搭建棚架, 加盖防鸟网。

\subsection{6 收获和指标测量}

2014年9月17日收获所有植株。收获时, 剪开栽 培袋, 用水洗去根上的土壤, 尽最大可能保持地上 和地下部分完整。植株自然风干后, 从茎基部剪断, 测量株高 $(\mathrm{H})$ 、穗长 $(\mathrm{EL})$ 。植株分成根、茎、叶、穗, 分别测量生物量。在植物进化和农业选择的过程中, 作物的种子大小趋向于较小的变化, 而种子的数量
一般具有很大的可变性(Sadras，2007)，据此，本实 验通过称量千粒种子质量和查数种子数量的方法估 算种子的生物量。由于谷穗上种子过多, 难以直接 一一查数, 故先查数穗轴上总分枝数, 然后从穗基 部、中部和尾部分别随机抽取1分枝, 计算各分枝的 小穗数, 从而估算谷穗上种子总数量, 进而估算单 株谷子所结的种子生物量(SB)。由于实验期间该地 区风较大, 收集凋落的叶片存在较大的难度, 故称 量单个植株完整叶片质量, 通过平均单片完整叶片 质量和查数单株总叶数的方法来估算单株谷子叶生 物量(LB)。

\section{3 数据处理}

数据均采用SPSS 17.0软件进行处理。采用三因 素方差分析方法, 检验邻株身份及其与种植密度和 土壤养分水平的交互作用对谷子茎生物量的影响。 为了避免植株大小及其相关的个体发育漂变 (ontogenetic drift)干扰谷子植株表型对邻株身份及 其与种植密度和土壤养分水平的交互作用的响应 (Weiner, 2004), 以茎生物量作为协变量, 通过协方 差分析来检验邻株身份及其与种植密度和土壤养分 水平的交互作用对谷子叶生物量、种子生物量、根 生物量、株高和穗长的影响, 其中为了使数据满足 方差齐性, 分析前对茎生物量、叶生物量和穗长数 据进行了自然对数转换。参照Cahill (2003)、Murphy 和Dudley (2009)、Lepik等(2012)的方法, 通过比较 
表1 邻株身份(亲缘株、非亲缘株、陌生株)及其与种植密度(高、低)和土壤养分(高、低)交互作用对谷子形态学特征和生物量分配的影响的协方差分 析(以茎生物量作为协变量)

Table 1 Analysis of covariance (using stem biomass as a covariate) for the morphology and biomass allocation of Setaria italica in response to neighbor identity (kin, non-kin or stranger neighbors) and its interaction with plant density (high or low) and soil nutrient level (high or low)

\begin{tabular}{|c|c|c|c|c|c|c|c|c|c|c|c|c|c|}
\hline \multirow{2}{*}{$\begin{array}{l}\text { 变异来源 } \\
\text { Source of variation }\end{array}$} & \multirow[t]{2}{*}{$d f$} & \multicolumn{2}{|c|}{ STB } & \multicolumn{2}{|c|}{ LBA } & \multicolumn{2}{|c|}{ SBA } & \multicolumn{2}{|c|}{ RBA } & \multicolumn{2}{|c|}{$\mathrm{H}$} & \multicolumn{2}{|c|}{ EL } \\
\hline & & $F$ & $P$ & $F$ & $P$ & $F$ & $P$ & $F$ & $P$ & $F$ & $P$ & $F$ & $P$ \\
\hline $\mathrm{D}$ & 1 & 129.80 & 0.000 & 0.32 & 0.573 & 15.66 & 0.000 & 12.15 & 0.001 & 13.98 & 0.000 & 5.42 & 0.022 \\
\hline SN & 1 & 12.74 & 0.001 & 7.61 & 0.007 & 43.24 & 0.000 & 5.48 & 0.022 & 0.02 & 0.878 & 47.99 & 0.000 \\
\hline NI & 2 & 10.82 & 0.000 & 5.76 & 0.004 & 4.53 & 0.013 & 0.19 & 0.828 & 5.54 & 0.005 & 0.32 & 0.725 \\
\hline $\mathrm{D} \times \mathrm{SN}$ & 1 & 0.04 & 0.850 & 0.40 & 0.530 & 3.03 & 0.084 & 0.01 & 0.920 & 12.29 & 0.001 & 4.49 & 0.036 \\
\hline $\mathrm{D} \times \mathrm{NI}$ & 2 & 3.53 & 0.032 & 4.15 & 0.018 & 0.68 & 0.508 & 0.20 & 0.817 & 2.42 & 0.093 & 0.84 & 0.432 \\
\hline $\mathrm{SN} \times \mathrm{NI}$ & 2 & 0.35 & 0.708 & 6.72 & 0.002 & 1.76 & 0.176 & 0.97 & 0.382 & 2.59 & 0.079 & 4.76 & 0.010 \\
\hline $\mathrm{D} \times \mathrm{SN} \times \mathrm{NI}$ & 2 & 0.19 & 0.828 & 3.63 & 0.029 & 2.27 & 0.107 & 0.40 & 0.669 & 1.75 & 0.177 & 3.78 & 0.026 \\
\hline STB & 1 & & & 195.88 & 0.000 & 54.96 & 0.000 & 64.39 & 0.000 & 161.32 & 0.000 & 12.61 & 0.001 \\
\hline
\end{tabular}

STB, 茎生物量; LBA, 叶生物量分配; SBA, 种子生物量分配; RBA, 根生物量分配; H, 株高; EL, 穗长; D, 种植密度; SN, 土壤养分水平; NI, 邻株身 份。加粗数据表示显著性值。

STB, stem biomass; LBA, leaf biomass allocation; SBA, seed biomass allocation; RBA, root biomass allocation; H, height; EL, ear length; D, plant density; SN, soil nutrient level; NI,neighbor identity. Boldface indicates significant values.

以茎生物量作为协变量的模型预测的各性状平均值 土标准误差的差异，来检验不同处理中谷子各部分 生物量分配、株高(茎伸长)和穗长的差异。

\section{2 结果和分析}

\section{1 邻株身份对谷子性状的影响}

邻株身份对谷子根生物量分配和穗长没有显著 影响, 而对茎生物量、叶生物量分配、种子生物量 分配和株高有显著影响(表1)。亲缘组谷子茎生物量 显著大于非亲缘组和陌生组, 后两者间没有显著差 异(图2A)。与此相反, 亲缘组谷子叶生物量分配显 著小于非亲缘组和陌生组, 后两者间也无显著差异 (图2B)。亲缘组和非亲缘组谷子种子生物量分配都 显著小于陌生组, 而两者间无差异(图2C)。非亲缘 组谷子株高最大, 显著大于陌生组, 但和亲缘组间 差异不显著(图2D)。

\section{2 邻株身份和种植密度交互作用对谷子性状的 影响}

邻株身份和种植密度交互作用对谷子种子生物 量分配、根生物量分配、株高和穗长均无显著影响, 对茎生物量和叶生物量分配有显著影响(表1)。在高 种植密度条件下, 谷子茎生物量和叶生物量分配在 各邻株身份处理组间无显著差异(图3A、3B)。在低 种植密度条件下, 亲缘组谷子茎生物量显著大于非 亲缘组和陌生组, 后两者间无显著差异(图3A), 与 此相反, 亲缘组谷子叶生物量分配显著小于非亲缘 组和陌生组, 后两者间也无显著差异(图3B)。随着
种植间距的增大(种植密度减小), 各邻株身份处理 组谷子茎生物量均显著增加, 而亲缘组谷子叶生物 量分配显著减少, 非亲缘组和陌生组叶生物量分配 在高、低种植密度条件下均无显著差异(图3A、3B)。

\section{3 邻株身份和土壤养分的交互作用对谷子性状 的影响}

邻株身份和土壤养分的交互作用对谷子茎生物 量、种子生物量分配、根生物量分配和株高无显著 影响, 对叶生物量分配和穗长有显著影响(表1)。在 低养分处理条件下, 非亲缘组谷子叶生物量分配与 亲缘组和陌生组间均无显著差异, 在高养分处理条 件下, 非亲缘组谷子叶生物量分配显著大于亲缘组 和陌生组(图4A)。在低土壤养分处理条件下, 亲缘 组谷子穗长显著小于非亲缘和陌生组, 后两者间无 显著差异, 而在高土壤养分处理条件下, 亲缘组谷 子穗长显著大于非亲缘组和陌生组, 后两者间仍无 显著差异(图4B)。

\section{3 讨论}

根、茎、叶生物量的变化、分枝数目、植物高 度及茎长度等可以响应因其他植物存在而表现出的 表型可塑性性状, 也可能是影响邻株适合度的竞争 性状, 因此也更有可能成为识别亲缘的指示性状 (Murphy \& Dudley, 2009; File et al., 2012)。植物的叶 是地上部分获取资源的器官, 叶生物量分配是衡量 植物地上部分竞争能力的一个很好的指标(Murphy \& Dudley, 2009)。研究发现, 和与非亲缘株为邻相 

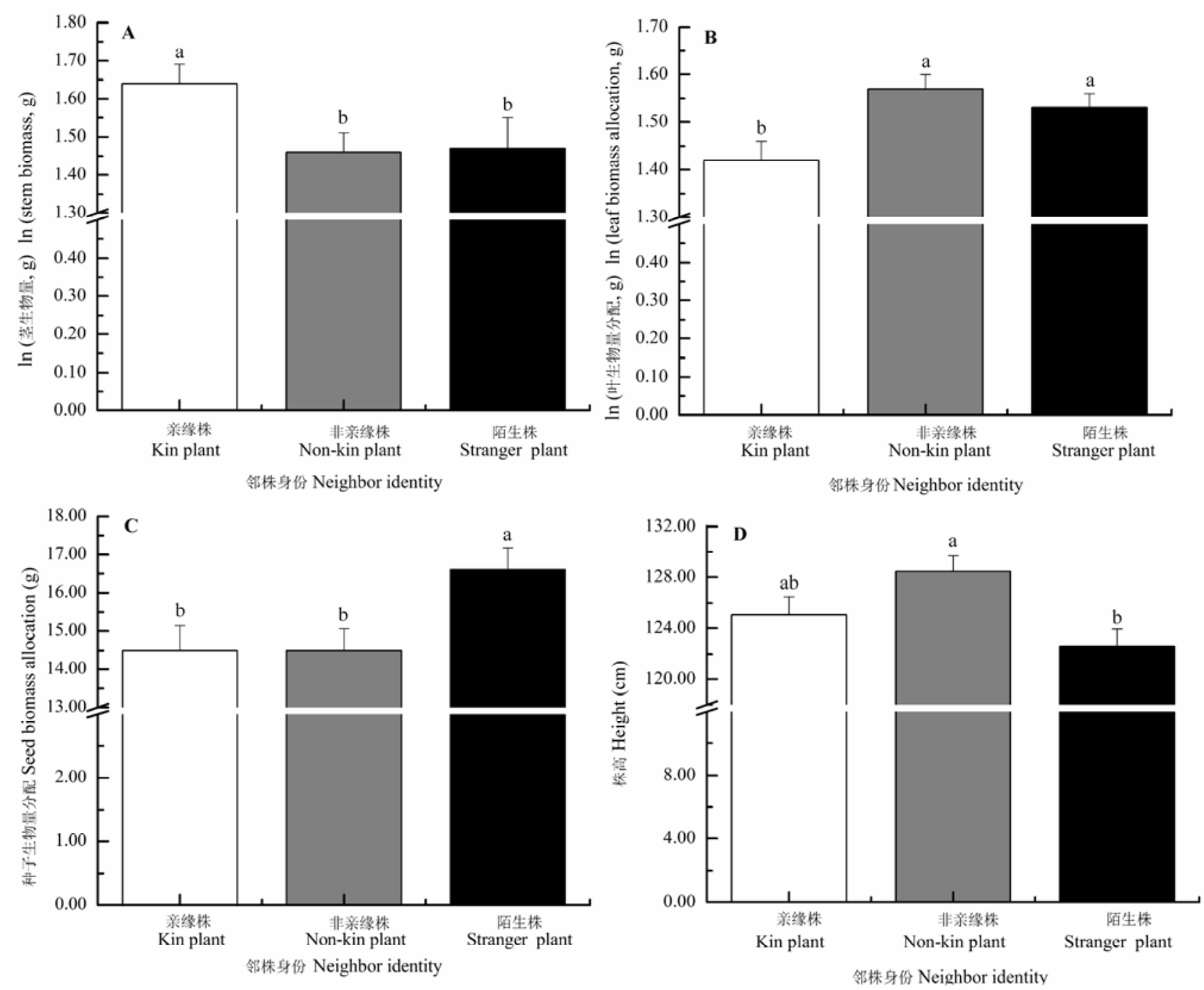

图2 邻株身份对谷子形态学特征和生物量分配的影响(平均值土标准误差)。谷子性状(除茎生物量)通过以茎生物量作为协变 量的模型预测得到，以此消除茎大小对各性状差异的影响。不同字母表示不同处理间差异显著 $(p<0.05, L S D)$ 。

Fig. 2 Effects of neighbor identity on the morphology and biomass allocation of Setaria italica (mean \pm SE). The traits (except for stem biomass) were predicted from the models with stem biomass as a covariate, by which the effects of the differences of stem biomass on trait means were accounted for. Different letters indicated significant differences among different treatments $(p<0.05, L S D)$.

比, 与亲缘株为邻时, Impatiens pallida植株显著减 少叶生物量分配(Murphy \& Dudley, 2009)。当拟南 芥(Arabidopsis thaliana)的邻株属于同一生态型时, 其叶片在水平方向上重新改变方向, 以减少邻株叶 片间相互重叠, 这一现象在邻株为不同生态型时并 没有发现(Crepy \& Casal, 2015)。本实验在根系隔离 的情况下 (避免地下部分竞争), 即在谷子地上部分 竞争占主导的情况下, 亲缘组谷子叶生物量分配显 著降低, 暗示着亲缘组谷子植株间显著减少了竞 争。这和地下部分竞争占主导作用时, 亲缘植株间 减少根生物量分配, 以减少彼此的干扰和竞争的结 果相似。植物增加株高(或茎生长), 是对弱光或遮阴
的响应方式之一, 以此增加植物竞争力(Weinig, 2000; Casal, 2013)。本实验中，亲缘组植株显著增加 茎生物量, 但株高小于非亲缘组, 即亲缘组植株茎 显著增粗。农作物茎秆粗壮有助于防倒伏, 因此可 能是对当地多风气候的一种适应。可见, 谷子响应 不同亲缘邻株的方式与谷子所处的生态环境(经过 人工驯化和种植, 主要面临地上竞争)存在一定的 协调性。类似的情况在Cakile edentula (Dudley \& File, 2007; Bhatt et al., 2011)、Impatiens pallida (Murphy \& Dudley, 2009)、拟南芥(Biedrzycki et al., 2010)和Ipomoea hederacea (Biernaskie, 2011)中也有 发现。由此, 本实验中, 亲缘组谷子植株间显著减少 


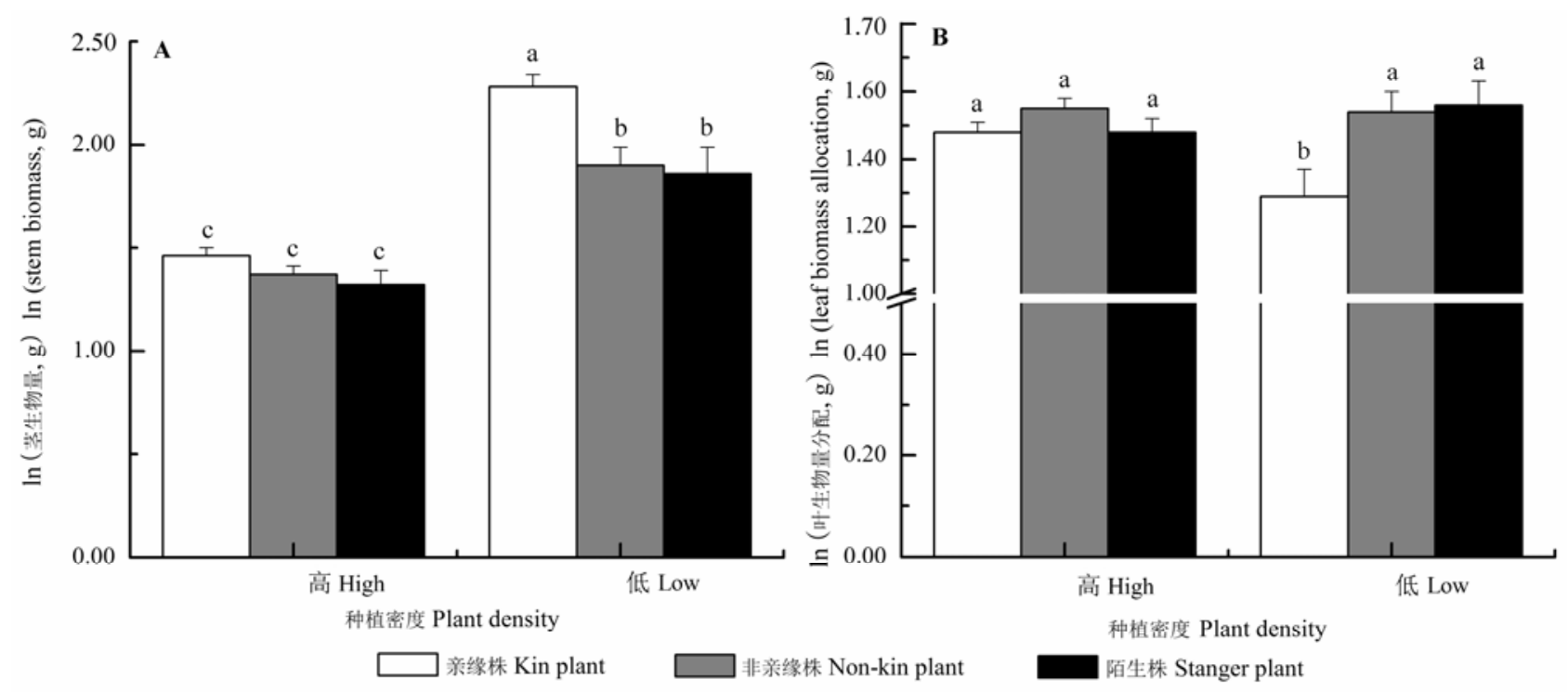

图3 邻株身份和种植密度的交互作用对谷子形态学特征和生物量分配的影响(平均值土标准误差)。谷子性状 (除茎生物量)通 过以茎生物量作为协变量的模型预测得到, 以此消除茎大小对各性状差异的影响。不同字母表示不同处理间差异显著 $(p<0.05, L S D)$ 。

Fig. 3 Interaction effects of neighbor identity and plant density on the morphology and biomass allocation of Setaria italica (mean \pm $S E$ ). The traits (except for stem biomass) were predicted from the models with stem biomass as a covariate, by which the effects of the differences of stem biomass on the trait means were accounted for. Different letters indicated significant differences among different treatments $(p<0.05, L S D)$.
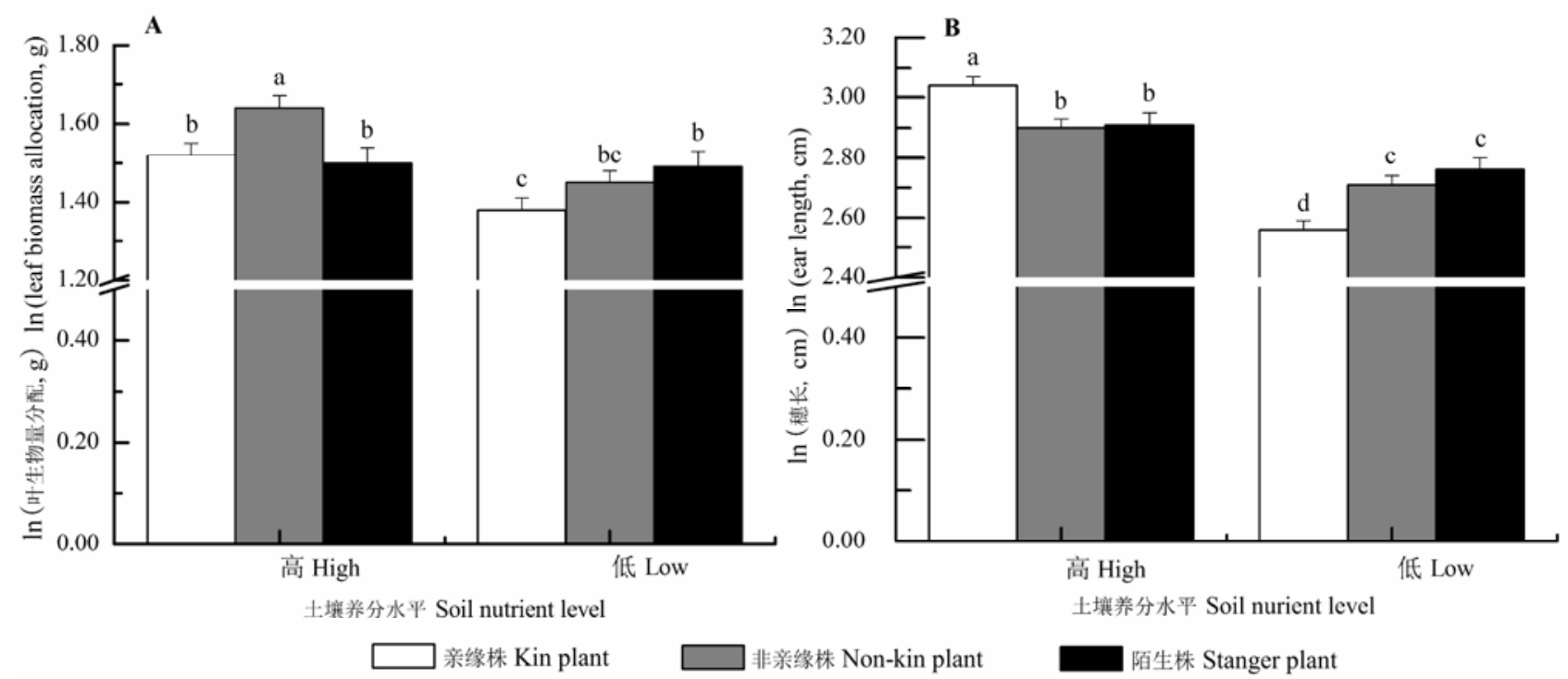

图4 邻株身份和土壤养分水平的交互作用对谷子形态学特征和生物量分配的影响(平均值土标准误差)。谷子性状 (除茎生物 量)通过以茎生物量作为协变量的模型预测得到, 以此消除茎大小对各性状差异的影响。不同字母表示不同处理间差异显著 $(p$ $<0.05, L S D)$ 。

Fig. 4 Interaction effects of neighbor identity and soil nutrient level on the morphology and biomass allocation of Setaria italica (mean $\pm S E$ ). The traits (except for stem biomass) were predicted from the models with stem biomass as a covariate, by which the effects of the differences of stem biomass on trait means were accounted for. Different letters indicated significant differences among different treatments $(p<0.05, L S D)$.

叶生物量分配(以此减少竞争), 茎秆增粗(以此增强 对当地多风气候的适应), 暗示着谷子可以识别亲 缘邻株并互助。而非亲缘组谷子植株, 显著增加叶
生物量分配, 显著降低茎生物量, 且和陌生组植株 相比显著增加株高，表明非亲缘谷子植株间竞争较 强。Crepy和Casal (2015)发现与邻株为不同生态型

www.plant-ecology.com 
的情况相比, 邻株为同生态型(亲缘近)的拟南芥植 株通过叶片的行为表现出亲缘互助, 但并没有表现 出更大的适合度。这个结果也在本实验中得到证实, 亲缘组和非亲缘组间谷子种子生物量分配无差异。 因此也在一定程度上说明了亲缘识别不一定导致亲 缘选择(File et al., 2012; Dudley et al., 2013)。另外, 由于适合度的指标受到亲缘选择、竞争、生态位分 化、频率依赖性选择等多个过程的影响, 这些过程 可能使得在竞争性状上表现出的亲缘识别现象在适 合度水平上无法表现, 因此也在一定程度上佐证了 适合度指标可能存在局限性的说法(File et al., 2012; Dudley et al., 2013)。

本实验中, 陌生组谷子种子生物量分配显著大 于亲缘组和非亲缘组, 可能主要是由于来自邻株 (糜)的竞争小, 谷子得以将更多的生物量投资于繁 殖部分。陌生组中的糜植株在6月底几乎都已完成抽 穗, 但收获时结实率低。另外, 陌生组谷子和糜植株 株高差距大(谷子平均高度 $122.55 \mathrm{~cm}$, 糜平均高度 $76.16 \mathrm{~cm}, p<0.001$, 未发表数据), 植株大小的差异 将导致不对称竞争, 影响竞争结果, 其中个体大的 植株占据优势(Bhatt et al., 2011)。更高的植株意味 着陌生组中的谷子可以获得更多的光照, 同时对糜 产生遮阴(Dudley et al., 2013), 并进一步限制其生 长, 以此增强竞争, 并将更多的生物量投资于繁殖, 增加适合度。

胁迫梯度假说预测，在严酷的环境下，植物间 更趋向于互助(Bertness \& Callaway, 1994; Maestre et al., 2009)。目前, 关于亲缘识别对环境因子的响 应方面的研究十分有限。多数的亲缘识别研究在室 内控制条件下进行, 而且多以一年生草本植物为研 究对象, 实验周期短, 种植间距小, 主要关注地下 部分竞争, 充分供给水、肥以消除由于根对资源的 竞争干扰实验结果(Dudley \& File, 2007; Murphy \& Dudley, 2009; Biedrzycki et al., 2010; Bhatt et al., 2011)。少数的研究表明, 植物亲缘识别受种植密度 制约, 在高密度(植株间距5 cm)种植条件下, Trifolium repens才表现出亲缘识别(Lepik et al., 2012)。

但Lupinus angustifolius在种植间距 $2 \mathrm{~cm}$ 和 $10 \mathrm{~cm}$ 时, 亲缘组和非亲缘组个体和群体水平上的适合度均没 有显著变化(Milla et al., 2009)。而拟南芥同生态型 植株在相邻间距10 cm时表现出亲缘识别(Crepy \& Casal, 2015)。亲缘识别对种植密度的响应可能因种
而异，同时受到植物生态、生活史阶段的影响。在 本实验中, 低密度有利于谷子亲缘识别的表达。植 物地下部分的竞争包括对资源的竞争和植物根系间 的相互干扰。有研究表明, 营养的可利用性水平显 著影响植物根系的竞争及生长(Markham \& Halwas, 2011), 甚至营养的供给方式也会影响植物生长 (Murphy et al., 2013)。植物能整合生境内的资源分 布格局和邻株的综合信息来决定根系的分布(Cahill et al., 2010), 因此, 土壤养分水平也可能影响植物 亲缘识别的表达。本实验中, 在高土壤养分条件下, 亲缘组谷子植株显著降低叶生物量分配, 且显著增 加穗长, 因此, 高土壤养分条件有利于谷子表达亲 缘识别能力。

由于本实验在自然条件下进行, 可能存在一些 不可控制的因素影响实验结果, 但结合该领域现有 的相关文献(这些文献基本沿用与本文一致的方法), 我们认为, 亲缘识别是本实验中亲缘组和非亲缘组 谷子植株形态学特征和生物量分配差异的一个很好 的解释, 即在根系隔离的情况下(即排除地下部分 相互作用), 谷子能够识别亲缘邻株, 暗示着地上部 分的竞争信号在谷子亲缘识别过程中扮演重要角 色。以往的研究较多关注地下部分竞争信号在植物 亲缘识别中的作用, 其实在这样的情况下, 也不可 避免地受到地上部分竞争信号的影响, 况且植物地 上部分的竞争信号也可以影响地下部分的竞争 (Gundel et al., 2014)。可见, 以往对植物地上部分竞 争信号在其亲缘识别中的作用被忽略和低估了。在 今后的研究中, 定量化地上和地下部分竞争信号在 诱导植物亲缘识别过程中所起的作用, 将是一个十 分重要但又充满挑战的研究方向。与胁迫梯度假说 的预测有所不同, 本实验结果说明了较低种植密度 和较高土壤养分有利于谷子亲缘识别能力的表达。

基金项目 国家自然科学基金(31170387和31370435)。

\section{参考文献}

Axelrod R, Hamilton WD (1981). The evolution of cooperation. Science, 211, 1390-1396.

Bais HP (2015). Shedding light on kin recognition response in plants. New Phytologist, 205, 4-6.

Bertness MD, Callaway RM (1994). Positive interactions in communities. Trends in Ecology and Evolution, 9, 191-193.

Bhatt MV, Khandelwal A, Dudley SA (2011). Kin recognition, 
not competitive interactions, predicts root allocation in young Cakile edentula seedling pairs. New Phytologist, 189, 1135-1142.

Biedrzycki ML, Bais HP (2010). Kin recognition in plants: A mysterious behaviour unsolved. Journal of Experimental Botany, 61, 4123-4128.

Biedrzycki ML, Jilany TA, Dudley SA, Bais HP (2010). Root exudates mediate kin recognition in plants. Communicative \& Integrative Biology, 3, 28-35.

Biernaskie JM (2011). Evidence for competition and cooperation among climbing plants. Proceedings of the Royal Society B: Biological Sciences, 278, 1989-1996.

Brooker RW, Maestre FT, Callaway RM, Lortie CL, Cavieres LA, Kunstler G, Liancourt P, Tielbörger K, Travis JMJ, Anthelme F, Armas C, Coll L, Corcket E, Delzon S, Forey E, Kikvidze Z, Olofsson J, Pugnaire F, Quiroz CL, Saccone P, Schiffers K, Seifan M, Touzard B, Michalet R (2008). Facilitation in plant communities: The past, the present, and the future. Journal of Ecology, 96, 18-34.

Cahill JF (2003). Lack of relationship between below-ground competition and allocation to roots in 10 gressland species. Journal of Ecology, 91, 532-540.

Cahill JF Jr, McNickle GG, Haag JJ, Lamb EG, Nyanumba SM, St Clair CC (2010). Plants integrate information about nutrients and neighbors. Science, 328, 1657-1657.

Callaway RM (2007). Positive Interactions and Interdependence in Plant Communities. Springer, Dordrecht, The Netherlands.

Casal JJ (2013). Photoreceptor signaling networks in plant responses to shade. Annual Review of Plant Biology, 64, 403-427.

Cavieres LA, Peñaloza A (2012). Facilitation and interference at the intraspecific level: Recruitment of Kageneckia angustifolia D. Don (Rosaceae) in the montane sclerophyllous woodland of central Chile. Perspectives in Plant Ecology, Evolution and Systematics, 14, 13-19.

Chen BJW, During HJ, Anten NPR (2012). Detect thy neighbor: Identity recognition at the root level in plants. Plant Science, 195, 157-167.

Cheplick GP (1992). Sibling competition in plants. Journal of Ecology, 80, 567-575.

Clara Castellanos M, Donat-Caerols S, González-Martínez SC, Verdú M (2014). Can facilitation influence the spatial genetics of the beneficiary plant population? Journal of Ecology, 102, 1214-1221.

Crepy MA, Casal JJ (2015). Photoreceptor-mediated kin recognition in plants. New Phytologist, 205, 329-338.

Dudley SA, File AL (2007). Kin recognition in an annual plant. Biology Letters, 3, 435-438.

Dudley SA, Murphy GP, File AL (2013). Kin recognition and competition in plants. Functional Ecology, 27, 898-906.

Fajardo A, McIntire EJB (2011). Under strong niche overlap conspecifics do not compete but help each other to sur- vive: Facilitation at the intraspecific level. Journal of Ecology, 99, 642-650.

Fang S, Clark RT, Zheng Y, Iyer-Pascuzzi AS, Weitz JS, Kochian LV, Edelsbrunner H, Liao H, Benfey PN (2013). Genotypic recognition and spatial responses by rice roots. Proceedings of the National Academy of Sciences of the United States of America, 110, 2670-2675.

Farrer EC, Goldberg DE (2011). Patterns and mechanisms of conspecific and heterospecific interactions in a dry perennial grassland. Journal of Ecology, 99, 265-276.

File AL, Murphy GP, Dudley SA (2012). Fitness consequences of plants growing with siblings: Reconciling kin selection, niche partitioning and competitive ability. Proceedings of the Royal Society B: Biological Sciences, 279, 209-218.

Gundel PE, Pierik R, Mommer L, Ballare CL (2014). Competing neighbors: Light perception and root function. Oecologia, 176, 1-10.

Hamilton WD (1964a). The genetical evolution of social behaviour. I. Journal of Theoretical Biology, 7, 1-16.

Hamilton WD (1964b). The genetical evolution of social behaviour. II. Journal of Theoretical Biology, 7, 17-52.

Hess L, de Kroon H (2007). Effects of rooting volume and nutrient availability as an alternative explanation for root self/non-self discrimination. Journal of Ecology, 95, 241-251.

Lepik A, Abakumova M, Zobel K, Semchenko M (2012). Kin recognition is density-dependent and uncommon among temperate grassland plants. Functional Ecology, 26, 1214-1220.

Li J, Sun G, Hu X, Zhang HX, Liu L, Wu N (2014). Kin selection in plants. Acta Ecologica Sinica, 34, 3827-3838. (in Chinese with English abstract) [李洁, 孙庚, 胡霞, 张洪 轩, 刘琳, 吴宁 (2014). 植物的亲缘选择. 生态学报, 34, 3827-3838.]

Liu B, Zhao M, Wang XR, Zhang LY, Zhao YK (2013). Effects of different nitrogen fertilizer rate on yield and economic benefits millet. Culture with Planting, (5), 11-12. (in Chinese with English abstract) [刘斌, 赵敏, 王显瑞, 张立 媛, 赵禹凯 (2013). 不同施氮量对谷子产量和效益的影 响. 耕作与栽培, (5), 11-12.]

Liu EK, Duan XS, Liu HX, Zhang DR, Liu YP, Liu H, Hou SL, Zhang M, Song YF, Wang XY, Zhou HZ (2013). The analysis of quantitative relationships between the planting density and yield in spring foxtail millet. Chinese Agricultural Science Bulletin, 29(30), 118-123. (in Chinese with English abstract) [刘恩魁, 段喜顺, 刘红霞, 张德荣, 刘 永平, 刘环, 侯升林, 张敏, 宋银芳, 王新玉, 周汉章 (2013). 春谷种植密度与产量的数量关系及其分析. 中 国农学通报, 29(30), 118-123.]

Maestre FT, Callaway RM, Valladares F, Lortie CJ (2009). Refining the stress-gradient hypothesis for competition and facilitation in plant communities. Journal of Ecology, 
97, 199-205.

Markham J, Halwas S (2011). Effect of neighbour presence and soil volume on the growth of Andropogon gerardii Vitman. Plant Ecology \& Diversity, 4, 265-268.

Mercer CA, Eppley SM (2014). Kin and sex recognition in a dioecious grass. Plant Ecology, 215, 845-852.

Milla R, Forero DM, Escudero A, Iriondo JM (2009). Growing with siblings: A common ground for cooperation or for fiercer competition among plants? Proceedings of the Royal Society B: Biological Sciences, 276, 2531-2540.

Milla R, Vélez Del Burgo A, Escudero A, Iriondo JM (2012). Kinship rivalry does not trigger specific allocation strategies in Lupinus angustifolius. Annals of Botany, 110, 165-175.

Mohana GS, Shaanker RU, Ganeshaiah KN, Dayanandan S (2001). Genetic relatedness among developing seeds and intra fruit seed abortion in Dalbergia sissoo (Fabaceae). American Journal of Botany, 88, 1181-1188.

Murphy GP, Dudley SA (2009). Kin recognition: Competition and cooperation in Impatiens (Balsaminaceae). American Journal of Botany, 96, 1990-1996.

Murphy GP, File AL, Dudley SA (2013). Differentiating the effects of pot size and nutrient availability on plant biomass and allocation. Botany, 91, 799-803.

Pakkasmaa S, Laurila A (2004). Are the effects of kinship modified by environmental conditions in Rana temporaria tadpoles? Annales Zoologici Fennici, 41, 413-420.

Sadras VO (2007). Evolutionary aspects of the trade-off between seed size and number in crops. Field Crops Research, 100, 125-138.
Semchenko M, Saar S, Lepik A (2014). Plant root exudates mediate neighbour recognition and trigger complex behavioural changes. New Phytologist, 204, 631-637.

Silvertown J (2004). Plant coexistence and the niche. Trends in Ecology \& Evolution, 19, 605-611.

Tonsor SJ (1989). Relatedness and intraspecific competition in Plantago lanceolata. The American Naturalist, 134, 897-906.

Weiner J (2004). Allocation, plasticity and allometry in plants. Perspectives in Plant Ecology, Evolution and Systematics, 6, 207-215.

Weinig C (2000). Differing selection in alternative competitive environments: Shade-avoidance responses and germination timing. Evolution, 54, 124-136.

Willson MF, Hoppes WG, Goldman DA, Thomas PA, KatusicMalmborg PL, Bothwell JL (1987). Sibling competition in plants: An experimental study. The American Naturalist, 129, 304-311.

Yang YJ, Wang HF, Guo PY, Wang YG, Yuan XY, Xing GF, Shao DH, Qi X, Xie LL, Nie ME, Guo J, Ning N (2013). Effects of fertilization and density on photosynthetic characteristics and yield of hybrid foxtail millet. Plant Nutrition and Fertilizer Science, 19, 566-576. (in Chinese with English abstract) [杨艳君, 王宏富, 郭平毅, 王玉国, 原 向阳, 邢国芳, 邵东红, 祁祥, 解丽丽, 聂萌恩, 郭俊, 宁娜 (2013). 施肥和密度对张杂谷5 号光合特性及产量 的影响. 植物营养与肥料学报, 19, 566-576.]

责任编委: 李镇清 责任编辑: 李 敏 